\title{
Images of the month: Intrahepatic multiple low-signal lesions from Aeromonas salmonicida infection
}

\author{
Authors: Yang Yang, ${ }^{\mathrm{A}}$ Hui Liu, ${ }^{\mathrm{B}}$ Wei Hua, ${ }^{\mathrm{C}}$ Caiping Guo ${ }^{\mathrm{D}}$ and Yulin Zhang ${ }^{\mathrm{E}}$
}
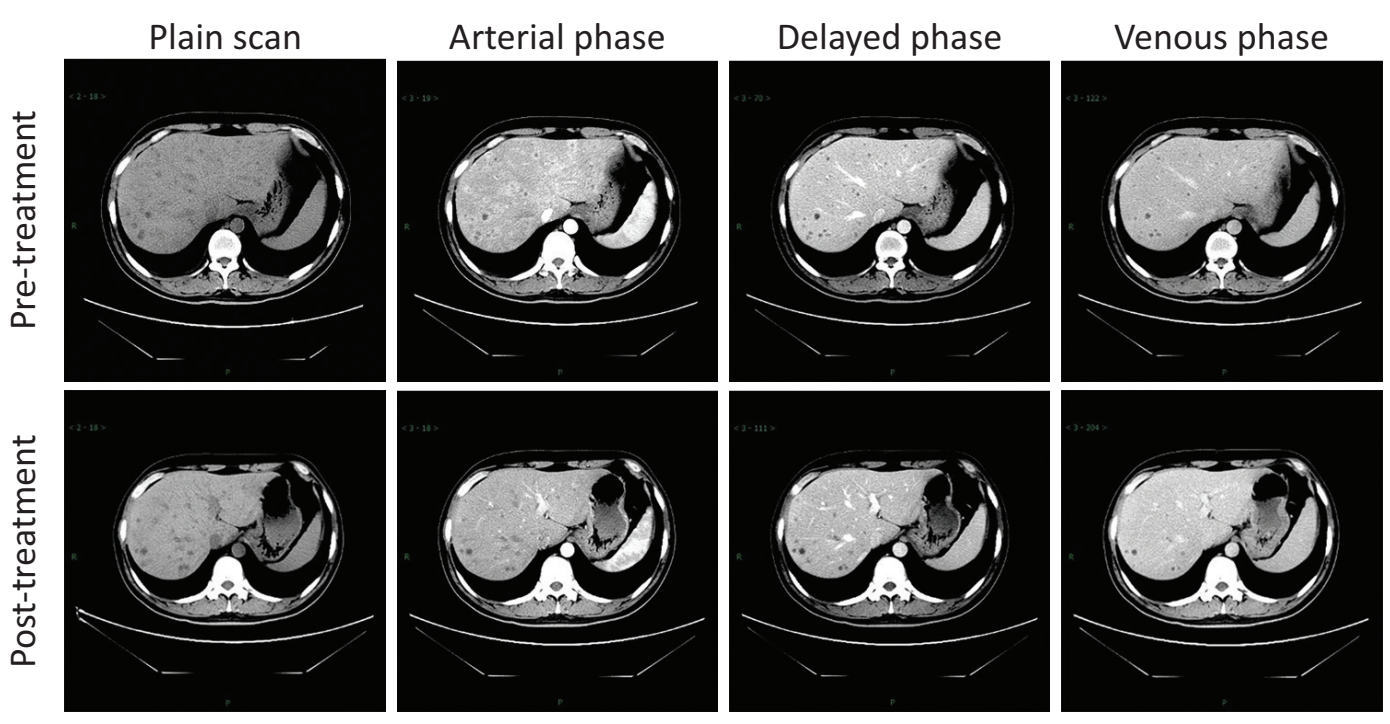

Fig 1. Computed tomography of the liver indicating multiple low-signal lesions in the liver before and after anti-Aeromonas salmonicida treatment.

KEYWORDS: Aeromonas salmonicida, liver infection, liver image, AIDS

DOI: $10.7861 /$ clinmed.2020-0144

\section{Case presentation}

A 28-year-old male, with HIV infection for 1 year, initially presented with 5 days of fever $\left(38.6^{\circ} \mathrm{C}\right)$ and mild abdomenal pain. He had been irregularly receiving antiretroviral therapy including lamivudine, tenofovir disoproxil fumarate and lopinavir/ ritonavir over the past year. The physical examination on admission was notable for tenderness in the epigastrium and pain on percussion in liver area. Laboratory tests indicated physical infections with white blood cell counts at $2.88 \times 10^{9} / \mathrm{L}$ and neutrophils counts at $1.58 \times 10^{9} / \mathrm{L}$, but multiple blood cultures did not reveal bacterial, fungi or mycobacterial growth. CD4 cell

Authors: ${ }^{\text {A }}$ resident, Beijing You'an Hospital, Beijing, China; ${ }^{B}$ chief physician, Beijing You'an Hospital, Beijing, China; ${ }^{C}$ associate chief physician, Beijing You'an Hospital, Beijing, China; ${ }^{D}$ chief physician, Beijing You'an Hospital, Beijing, China; ${ }^{E}$ Chief physician, Beijing You'an Hospital, Beijing, China counts were 292 cells/ $\mu$ L. Multiple low-signal lesions in the liver area were shown by computed tomography (CT; Fig 1, upper row). A lumbar puncture and cerebrospinal fluid analysis were performed to exclude the possibility of intracranial infection. The patient was suspected as having a liver abscess and empirical antibiotics were administrated including cefotaxime sodium plus sulbactam sodium and metronidazole, which could cover the common Escherichia coli and amoeba. But the symptoms of the patient did not alleviate. In order to find the cause of the disease, we performed a percutaneous liver needle biopsy and the histopathology revealed considerable neutrophil infiltration, which were consistent with inflammation in the liver tissue (Fig 2). But no pathogenic microorganism was found in liver tissue with specific stain and culture. Further metagenomic sequencing (MGS) detected Aeromonas salmonicida in the liver tissue as standard protocol, although the detected $A$ salmonicida reads were only four (Fig 3), and phylogenetic reconstruction showed each of the four sequences had a good homology with reference sequences (Fig 4). The patient then confessed eating half cooked fish before he was sick. Based on this finding, empirical antibiotics were conversed to moxifloxacin that $\operatorname{targets} A$ salmonicida and the patient rapidly recovered to his premorbid status, although liver lesions did not completely disappear in CT (Fig 1, lower row). One month later, second liver needle biopsy and MGS did not find $A$ salmonicida infection again. 


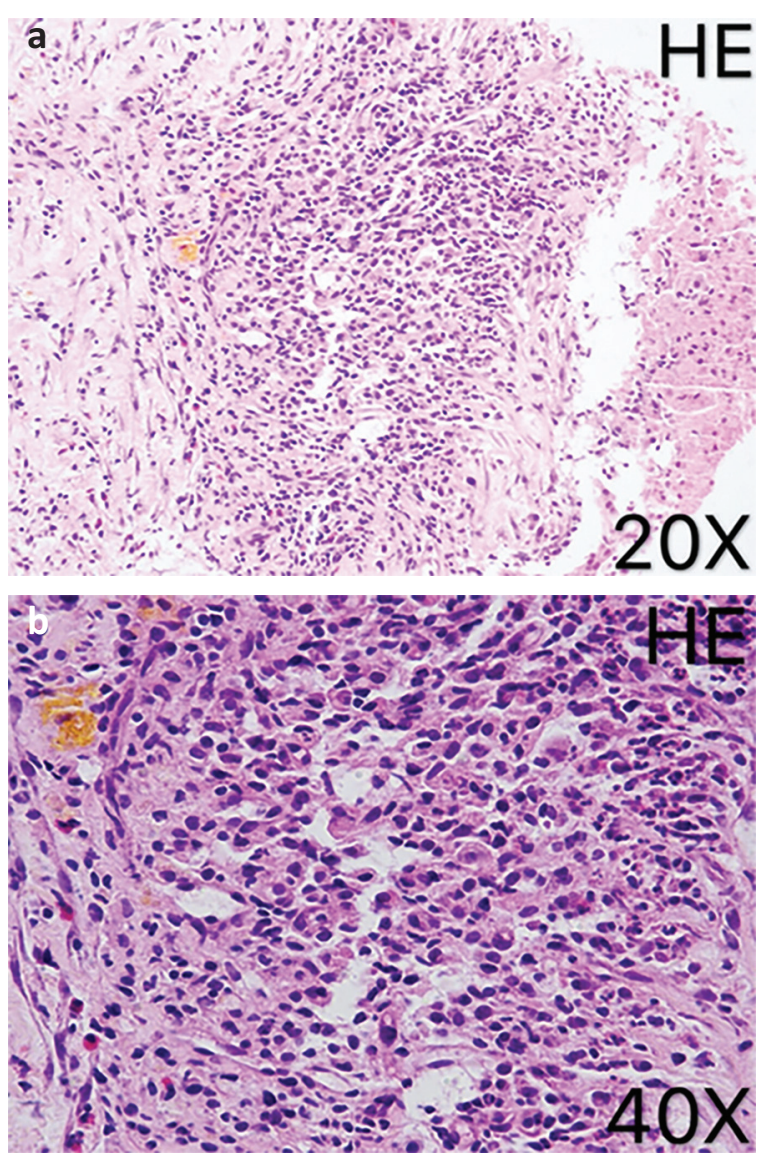

Fig 2. Haematoxylin and eosin stain of the liver biopsy specimen revealing considerable neutrophil infiltration consistent with inflammation.

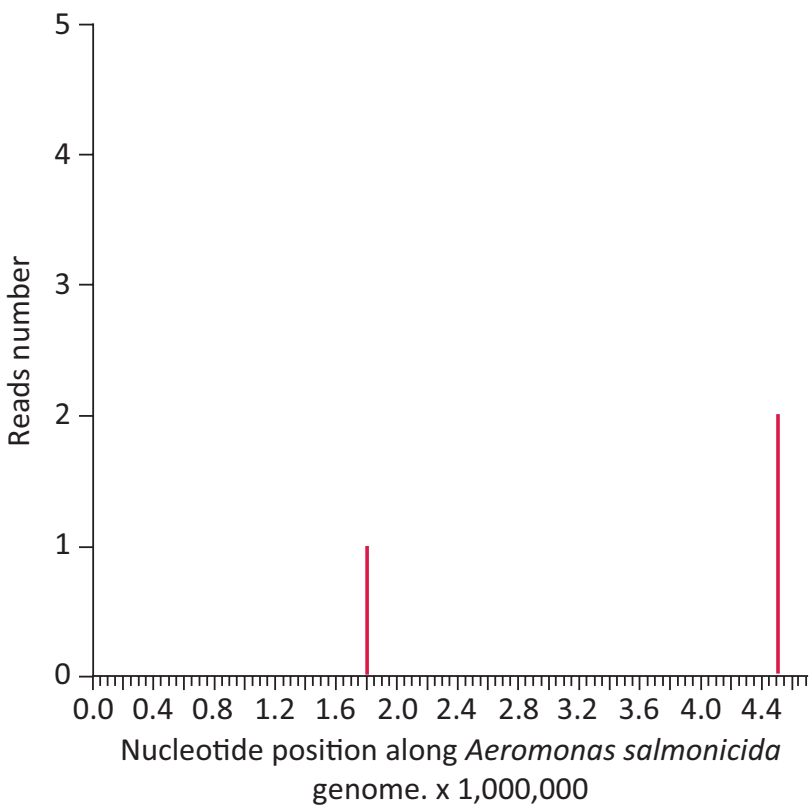

Fig 3. Nucleotide position chromosome coverage of metagenomic sequencing detecting Aeromonas salmonicida genome. Aeromonas salmonicida $0.0115 \%$ total coverage.

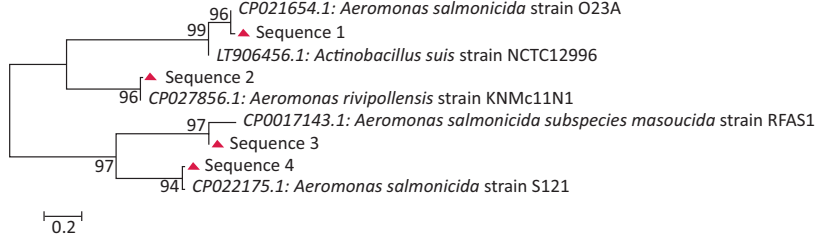

Fig 4. Phylogenetic tree constructed by the neighbour-joining method and Kimura two-parameter model and evaluated by the bootstrap resampling method using 1,000 replicates as implemented in Mega 5.0 software. Five reference sequences were obtained from GenBank. The numbers of the phylogenetic tree denoted the reliability of phylogenetic analysis. The scale bar represents genetic distance ( 0.2 substitution per site).

\section{Discussion}

A salmonicida is a member of vibrio family and is a rare multi-host pathogen which can infect immunocompromised individuals, mainly resulting in gastroenteritis and sepsis. ${ }^{1}$ Human liver infection of $A$ salmonicida has rarely been reported, so its characteristic image performance is not clear. ${ }^{2} \mathrm{MGS}$ is a type of DNA sequencing technology which mainly targets a person's genome for exceptional genetic disorders and detects rare pathogen infection when traditional tests are negative or quicker result judgement could increase patients' survival rate. ${ }^{3,4}$ However, the high sensitivity of MGS sometimes results in false-positive results, which requires the clinician to accurately judge the detection report according to the clinical diagnosis pathway for specific disease. ${ }^{5}$ It is reasonable that an AIDS patient with fever of unknown origin and liver low-signal lesions are conventionally suspected as having liver infection even if no pathogen diagnosis evidence. As traditional blood pathogen culture and empirical antibacterial therapy did not benefit this patient, MGS played a pivotal role in providing a valuable diagnostic support.

\section{Funding}

The authors are supported by the National Natural Science Foundation of China (81873761) and the 13th Key Science and Technology Five Year Plan of China (2018ZX10302104).

\section{References}

1 Janda JM, Abbott SL. The genus Aeromonas: taxonomy, pathogenicity, and infection. Clin Microbiol Rev 2010;23:35-73.

2 Tsai MS, Kuo CY, Wang MC et al. Clinical features and risk factors for mortality in Aeromonas bacteremic adults with hematologic malignancies. J Microbiol Immunol Infect 2006:39:150-4.

3 Human Microbiome Project Consortium. A framework for human microbiome research. Nature 2012;486:215-21.

4 Pfeiffer $\mathrm{F}$, Zamora-Lagos MA, Blettinger $\mathrm{M}$ et al. The complete and fully assembled genome sequence of Aeromonas salmonicida subsp. pectinolytica and its comparative analysis with other Aeromonas species: investigation of the mobilome in environmental and pathogenic strains. BMC Genomics 2018;19:20.

5 Taylor JC, Martin HC, Lise S et al. Factors influencing success of clinical genome sequencing across a broad spectrum of disorders. Nat Genet 2015;47:717-26.

Address for correspondence: Dr Yulin Zhang, Department of Infectious Diseases, Beijing You'an Hospital, Capital Medical University, Beijing Institute of Hepatology, Beijing 100069, China.

Email: yulinzhang@ccmu.edu.cn 